\title{
The clinical implication of serum cyclophilin A in patients with chronic obstructive pulmonary disease
}

This article was published in the following Dove Press journal: International Journal of COPD

\author{
Ming Zhang' \\ Jingjing Tang' \\ Jiafeng Yin ${ }^{2}$ \\ Xiaoying Wang ${ }^{3}$ \\ Xiangli Feng' \\ Xia Yang' \\ Hu Shan' \\ Qiuhong Zhang' \\ Jie Zhang' \\ Yali Li' \\ 'Department of Respiratory and \\ Critical Care Medicine, The Second \\ Affiliated Hospital of Xi'an Jiaotong \\ University, Xi'an, ${ }^{2}$ Department of \\ Laboratory Examination, The Second \\ Affiliated Hospital of Xi'an Jiaotong \\ University, Xi'an, ${ }^{3} \mathrm{Health}$ Examination \\ Center, The Second Affiliated \\ Hospital of Xi'an Jiaotong University, \\ Xi'an, People's Republic of China
}

\section{Correspondence: Yali Li}

Department of Respiratory and Critical Care Medicine, The Second Affiliated Hospital of Xi'an Jiaotong University, No 157 West Fifth Road, Xi'an, Shaanxi 710004, People's Republic of China

Email 81276894@qq.com
Background: Cyclophilin A (CyPA) is a secreted molecule that is regulated by inflammatory stimuli. Although inflammation has an important role in the pathogenesis of chronic obstructive pulmonary disease (COPD), little is known regarding the relationship between serum CyPA and COPD.

Methods: Ninety-three COPD patients with acute exacerbation were enrolled in the study and were reassessed during the convalescence phase. Eighty-eight controls were matched for age, gender, body mass index, smoking index and comorbidity. The basic clinical information and pulmonary function of all participants were collected. Serum levels of CyPA and other inflammation indexes were further measured.

Results: Serum CyPA was significantly increased in convalescent COPD patients compared to healthy controls, and further elevated in COPD patients with acute exacerbation. Serum CyPA positively correlated with serum interleukin-6, matrix metalloproteinase- 9 and high-sensitivity C-reactive protein in both the exacerbation and convalescence phases of COPD. Furthermore, it negatively correlated with percent value of forced expiratory volume in 1 second $\left(\mathrm{FEV}_{1} \%\right)$ predicted and $\mathrm{FEV}_{1} /$ forced vital capacity in convalescent $\mathrm{COPD}$ patients.

Conclusion: These results suggest that serum CyPA can be used as a potential inflammatory biomarker for COPD and assessment of serum CyPA may reflect the severity of inflammation in COPD.

Keywords: cyclophilin A, chronic obstructive pulmonary disease, exacerbation, convalescence, inflammation

\section{Introduction}

Chronic obstructive pulmonary disease (COPD) is characterized by irreversible airflow limitation, chronic airway inflammation, and emphysema. The morbidity and mortality rates of COPD constantly increase partly because of environmental pollution and an aging population. ${ }^{1}$ It has become a challenging problem worldwide.

Several mechanisms have been involved in the pathogenesis of COPD, such as inflammation, oxidant-antioxidant imbalance, proteinase-antiproteinase imbalance, immunologic mechanisms, apoptosis, and ineffective repair. ${ }^{2}$ The inflammatory response has played an important role in the progression of COPD. ${ }^{3}$ Airway inflammation in COPD is characterized by the infiltration of T lymphocytes, macrophages, and neutrophils. ${ }^{4}$ It has been shown that airway inflammation correlates with disease severity and pulmonary function in COPD, as they are able to induce tissue damage through the release of various oxidants and proteases. ${ }^{5}$ Not only airway inflammation, but also systemic inflammation is now recognized as an integral part of COPD 
pathogenesis. ${ }^{6}$ Serum C-reactive protein, a well-known systemic inflammatory marker, has been demonstrated to correlate with disease severity, acute exacerbation frequency, and prognosis of COPD. ${ }^{7}$ It has also been reported that several other serum biomarkers are used to assess systemic inflammation during the clinical course of COPD. ${ }^{8}$

Cyclophilin A (CyPA) is a ubiquitously expressed protein belonging to the immunophilin family, which shares peptidyl-prolylcis-trans isomerase activity and was first identified as a cytosolic binding protein of the immunosuppressant cyclosporin A in 1984. ${ }^{9}, 10 \mathrm{CyPA}$ has been involved in a variety of physiologic and pathologic activities, including protein folding, trafficking, and T-cell activation. ${ }^{11}$ Although CyPA has initially demonstrated activity as an intracellular protein, it can be secreted into the extracellular environment in various cell types under inflammatory stimuli and oxidative stress. ${ }^{12}$ The increased serum levels of CyPA can be observed in patients with acute coronary syndrome, diabetes mellitus, and chronic kidney disease. ${ }^{13-15}$ Despite inflammation and oxidative stress playing important roles in the pathogenesis of COPD, little is known regarding the relationship between serum CyPA and COPD. Therefore, our present study was designed to investigate the alterations of serum CyPA during clinical recovery from COPD exacerbation as well as the correlations between serum CyPA and other inflammatory biomarkers.

\section{Materials and methods Reagents}

CyPA, high-sensitivity C-reactive protein (hsCRP), interleukin-6 (IL-6), and matrix metalloproteinase-9 (MMP-9) enzyme-linked immunosorbent assay (ELISA) kits were all purchased from Yuanye Biological Technology Co. Ltd (Shanghai, People's Republic of China). The detection limit of the kits is $0.1 \mathrm{ng} / \mathrm{mL}$ for CyPA, $0.1 \mathrm{mg} / \mathrm{L}$ for hsCRP, $1 \mathrm{pg} / \mathrm{mL}$ for IL-6, and $1 \mathrm{ng} / \mathrm{mL}$ for MMP-9.

\section{Patients and control subjects}

COPD patients with acute exacerbation phase were admitted to the Department of Respiratory and Critical Care Medicine of The Second Affiliated Hospital of Xi'an Jiaotong University, between January 2016 and December 2016. The diagnosis criteria of COPD and acute exacerbation phase were according to the Global Initiative for Chronic Obstructive Lung Disease. ${ }^{16}$ Patients who had asthma, bronchiectasis, pneumonia, sleep apnea syndrome, cancer, coronary artery disease, diabetes mellitus, and autoimmune diseases were excluded by medical history inquiry, physical examination, biochemical parameters determination (including autoantibodies and tumor markers), pulmonary function, electrocardiogram, ultrasonic cardiogram, and computed tomography. All enrolled patients were followed until the individual was medically stable enough to leave the hospital and not prone to acute exacerbation again. Patients who had adverse clinical outcomes were also excluded, including death from any cause in hospital, requiring mechanical ventilation and length of hospitalization time shorter than 1 day or longer than 30 days. Finally, 93 cases met the required inclusion and exclusion criteria, and were further evaluated 8 (interquartile range [IQR]: 7-11) days after the onset of exacerbation when they were convalescent. Healthy controls were recruited from the health examination center of the hospital, and 88 controls were matched with COPD patients for age, gender, body mass index, and smoking index. The present research was approved by the Research Committee of Human Investigation of Medical College of Xi' an Jiaotong University. All participants gave written informed consent.

\section{Treatments of COPD}

The routine treatments for exacerbated COPD during hospitalization were as follows: atomization inhalation of corticosteroids, $\beta_{2}$-agonists, and anticholinergics, and intravenous infusions of methylprednisolone (with an initial dose of $40 \mathrm{mg}$ daily), aminophylline, and expectorants. Antibiotics were administered if a bacterial infection was suspected and adjusted according to clinical symptoms and signs, sputum culture tests, and biochemical inflammatory markers. Oxygen was provided in order to maintain oxygen saturation $\geq 90 \%$. Nebulized bronchodilators were prescribed for maintenance therapy upon discharge from the hospital.

\section{Clinical variables}

Variables including demographic data, smoking habits, course of disease, comorbidity condition, and respiratory symptoms were recorded on the day of admission. In consideration of severe breathing difficulties, exacerbated COPD patients did not perform pulmonary function examination. When they were stable enough to perform the spirometer maneuver before leaving the hospital, pulmonary function was assessed by an experienced technician. The basic clinical information and pulmonary function of healthy controls were also collected.

\section{Sample collection and measurement}

Venous blood samples of COPD patients were collected at the beginning of hospitalization (exacerbation) and at the end of hospitalization (convalescence). In addition, samples of healthy controls were collected upon healthy examination. Blood samples were centrifuged at $1000 \times g$ at $4^{\circ} \mathrm{C}$ for 
$20 \mathrm{~min}$. And then serum was separated and stored at $-80^{\circ} \mathrm{C}$ until further analysis. Routine blood testing was conducted for determining white blood cells (WBC) and neutrophil\% by an autoanalyser (Sysmex XE-2100, Kobe, Japan) in the clinical laboratory. Serum concentrations of CyPA, hsCRP, IL-6, and MMP-9 were determined by commercial sandwich ELISA kits according to the manufacturer's instructions. All the samples from each participant were measured in the same assay to reduce interassay variability.

\section{Statistical analysis}

Statistical analyses were conducted with SPSS version 17.0 software (SPSS Inc., Chicago, IL, USA). All data were examined with Kolmogorov-Smirnov test for normal distribution. Continuous variables were presented as mean \pm SD or median (interquartile range) according to the distribution state. Categorical variables were presented as a percentage. Normally distributed data were analyzed using Student's $t$-tests, non-normally distributed data were compared using Wilcoxon-Mann-Whitney test, and categorical variables were compared with $\chi^{2}$ test. Correlation coefficients between CyPA levels and other laboratory values were analyzed by partial correlation with age, body mass index, and course of disease as continuous variables, and gender, smoking status, and presence of hypertension as dichotomous variables. Multivariate linear regression analysis was used to assess the relationship between CyPA and age, gender, body mass index, smoking status, course of disease, hypertension, and disease status. A value of $P<0.05$ was considered significant.

\section{Results}

Table 1 summarizes the clinical characteristics of all study participants. The groups were well matched with respect to

Table I Basic characteristics of the study population

\begin{tabular}{llll}
\hline Parameter & COPD & Control & P-value \\
\hline Number & 93 & 88 & \\
Age (years) & $63.9 \pm 7.29$ & $62.7 \pm 5.29$ & 0.232 \\
Gender (male \%) & 77.4 & 78.4 & 0.873 \\
Body mass index $\left(\mathrm{kg} / \mathrm{m}^{2}\right)$ & $23.4 \pm 3.76$ & $24.2 \pm 2.59$ & 0.107 \\
Never smokers $(\%)$ & 32.3 & 37.5 & 0.459 \\
Smoking index (pack-years)* & $46.8 \pm 23.0$ & $43.3 \pm 26.5$ & 0.447 \\
Hypertension (\%) & 18.3 & 15.9 & 0.672 \\
Course of disease (years) & $10(4.5-13.5)$ & $\mathrm{NA}$ & \\
Hospitalization time (days) & $8(7-1 \mathrm{I})$ & $\mathrm{NA}$ & \\
Medication & & & \\
$\quad$ Inhaled bronchodilators (\%) & 100 & 0 & $\mathrm{NA}$ \\
$\quad$ Inhaled corticosteroids (\%) & 55.9 & 0 & $\mathrm{NA}$ \\
\hline
\end{tabular}

Notes: Data are expressed as mean \pm SD or percentage or median (interquartile range). *Includes current and former smokers.

Abbreviations: COPD, chronic obstructive pulmonary disease; NA, not applicable. age, gender, body mass index, and smoking index. The proportion of subjects with hypertension was similar between COPD patients and healthy controls $(P=0.672)$. The course of COPD was 10 (IQR: 4.5-13.5) years and median hospitalization time of those patients with exacerbated COPD was 8 (IQR: 7-11) days.

Figure 1 shows the parameters of pulmonary function including forced vital capacity (FVC), forced expiratory volume in 1 second $\left(\mathrm{FEV}_{1}\right)$, and $\mathrm{FEV}_{1} / \mathrm{FVC}$ ratio. Compared to healthy controls, $\mathrm{FVC} \%$ predicted and $\mathrm{FEV}_{1} \%$ predicted in the convalescent COPD patients were both significantly decreased $(76.4 \pm 14.4$ versus $85.7 \pm 10.3$ and $46.1 \pm 9.92$ versus $90.4 \pm 10.0$, respectively, both $P<0.001)$. The ratio of $\mathrm{FEV}_{1} / \mathrm{FVC}$ in convalescent COPD patients was lower than that in the healthy control group $(45.8 \pm 10.4$ versus 89.3 $\pm 6.40, P<0.001)$.

Serum concentration of CyPA in COPD patients with exacerbation phase was significantly higher than in the convalescence phase $(4.97 \pm 0.68$ versus $4.02 \pm 0.63 \mathrm{ng} / \mathrm{mL}$, $P<0.001)$. Moreover, CyPA serum concentration in convalescent COPD patients was notably increased compared to healthy controls $(4.02 \pm 0.63$ versus $3.36 \pm 0.51 \mathrm{ng} / \mathrm{mL}$, $P<0.001$, Figure 2A). Serum levels of hsCRP were 10.2 (IQR: 9.47-11.3) $\mathrm{mg} / \mathrm{L}$ in exacerbated COPD, 7.86 (IQR: 7.20-8.80) $\mathrm{mg} / \mathrm{L}$ in convalescent COPD, and 6.89 (IQR: $6.07-7.68) \mathrm{mg} / \mathrm{L}$ in healthy controls, and all the differences were significant $(P<0.001$, Figure 2B). Serum concentrations of IL-6 and MMP-9 in the exacerbation phase of COPD were significantly higher than those in the convalescence phase $(39.6 \pm 5.81$ versus $28.1 \pm 4.93 \mathrm{pg} / \mathrm{mL}$ and $341 \pm 51.5$ versus $269 \pm 41.6 \mathrm{pg} / \mathrm{mL}$, respectively, both $P<0.001$ ). Compared to healthy controls, serum levels of IL- 6 and MMP-9 in the convalescent COPD patients were also higher $(28.1 \pm 4.93$ versus $21.5 \pm 4.13 \mathrm{pg} / \mathrm{mL}$ and $269 \pm 41.6$ versus $205 \pm 30.5 \mathrm{pg} / \mathrm{mL}$, respectively, both $P<0.001$, Figure $2 \mathrm{C}$ and $\mathrm{D}$ ). In addition, there was a significant decline in $\mathrm{WBC}$ and neutrophil\% from the exacerbation to the convalescence phase of COPD $(P=0.047$ and $P<0.001$, respectively). Furthermore, the values of WBC and neutrophil\% further decreased in healthy controls (both $P<0.001$, Figure 2E and F).

The partial correlation coefficients of serum CyPA with other laboratory parameters after adjustment for age, gender, body mass index, smoking index, course of disease, and comorbidity are presented in Tables 2 and 3. During the exacerbation and convalescence phases of COPD, serum CyPA values positively correlated with hsCRP ( $r=0.442$ and 0.441 , both $P<0.05)$, IL-6 ( $r=0.343$ and 0.307 , both $P<0.05$ ), and MMP-9 ( $r=0.749$ and 0.778 , both $P<0.05)$. Serum CyPA 
A

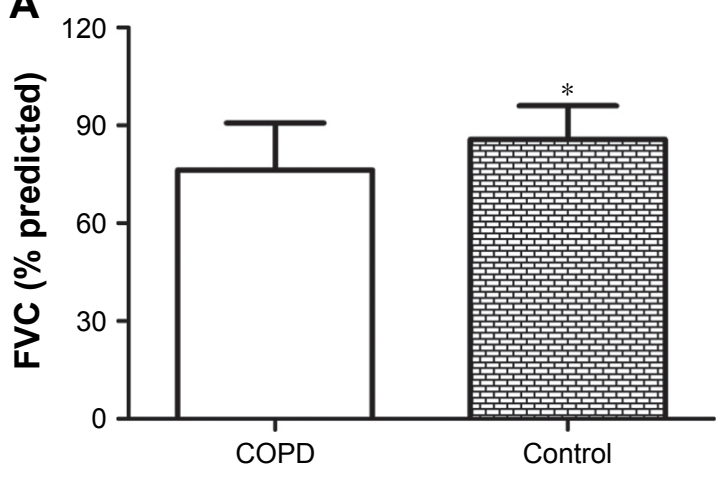

C

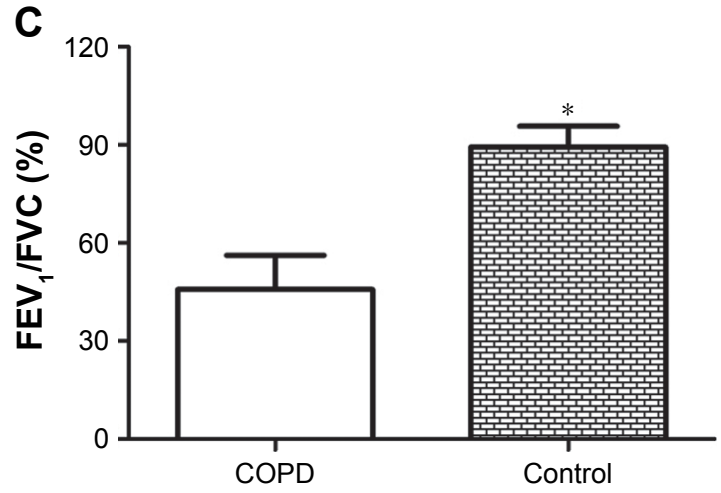

B

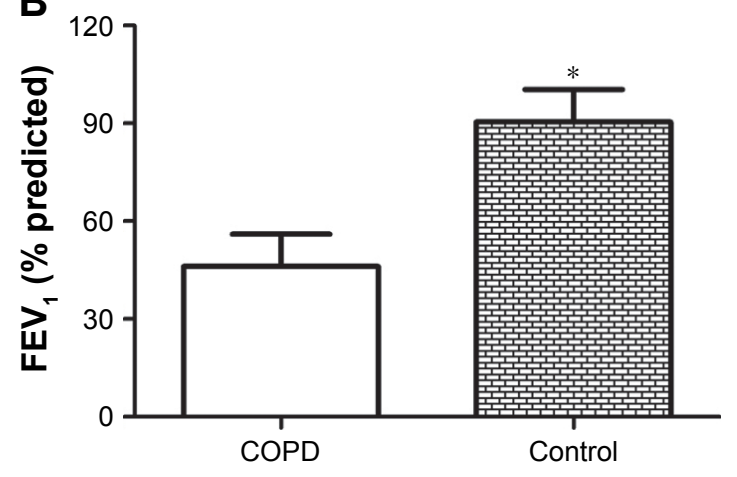

Figure I Pulmonary function of convalescent COPD patients $(n=93)$ and healthy controls $(n=88)$

Notes: The parameters of pulmonary function including FVC\% predicted $(\mathbf{A}), \mathrm{FEV}, \%$ predicted $(\mathbf{B})$ and $\mathrm{FEV} / \mathrm{FVC}(\mathbf{C})$ were collected. Data are mean \pm SD. $* P<0.05$. Abbreviations: COPD, chronic obstructive pulmonary disease; $\mathrm{FEV}_{1}$, forced expiratory volume in I second; FVC, forced vital capacity.

was also positively correlated with $\mathrm{WBC}$ and neutrophil\% in exacerbated COPD patients $(r=0.386$ and 0.254 , both $P<0.05$ ), but there were no statistical correlations between CyPA and WBC as well as neutrophil\% in convalescent COPD patients ( $r=0.197$ and 0.164 , both $P>0.05$ ). During the convalescence phase of COPD, there was no statistical correlation between CyPA and $\mathrm{FVC} \%$ predicted $(r=-0.154$, $P=0.154)$. In addition, CyPA negatively correlated with $\mathrm{FEV}_{1} \%$ predicted $(r=-0.271, P=0.011)$ and $\mathrm{FEV}_{1} / \mathrm{FVC}$ $(r=-0.291, P=0.006)$ in convalescent COPD patients.

Multivariate linear regression analysis with $\mathrm{CyPA}$ as the dependent variable showed that COPD disease status was the only major influence factor affecting CyPA (unadjusted $r^{2}=0.400$, adjusted $r^{2}=0.376$ ), while age, gender, body mass index, smoking status, course of disease, and hypertension did not appear to be significant factors influencing CyPA (Table 4).

\section{Discussion}

CyPA is evolutionarily well conserved and expressed in all cells of organisms in both prokaryotes and eukaryotes. ${ }^{17}$ It is mainly localized in the cytoplasm and shows a variety of intracellular functions, including intracellular signaling, protein trafficking, and the regulation of other protein activities. ${ }^{11}$ CyPA can also be secreted into the extracellular environment from various cell types in response to infection, hypoxia, and oxidative stress. ${ }^{18-20}$ However, the concrete mechanism by which CyPA is secreted from cells is still unclear and may be associated with the acetylation of CyPA. ${ }^{21}$ The secreted form of CyPA is known to function as an autocrine/paracrine factor, which mediates intercellular signal communication. ${ }^{22}$

Current studies have demonstrated that CyPA is involved in the pathogenesis of several human diseases, such as cardiovascular disease, rheumatoid arthritis, sepsis, viral infection, cancer, nervous system degeneration, asthma, and periodontitis. ${ }^{18,23-29}$ Proteomic analysis has found that the expression of CyPA is upregulated in smokers and particularly notable in COPD smokers. ${ }^{30}$ However, alterations of serum CyPA in COPD patients remain unclear. Our present study showed that serum CyPA levels were increased in convalescent COPD patients compared to healthy controls, and further elevated in COPD patients with acute exacerbation. The major reason for the upregulation of serum CyPA concentration in COPD patients may be due to the inflammatory stimuli and oxidative stress. In addition, it has been reported that extracellular CyPA binds to extracellular matrix metalloproteinase inducer (EMMPRIN, CD147) 

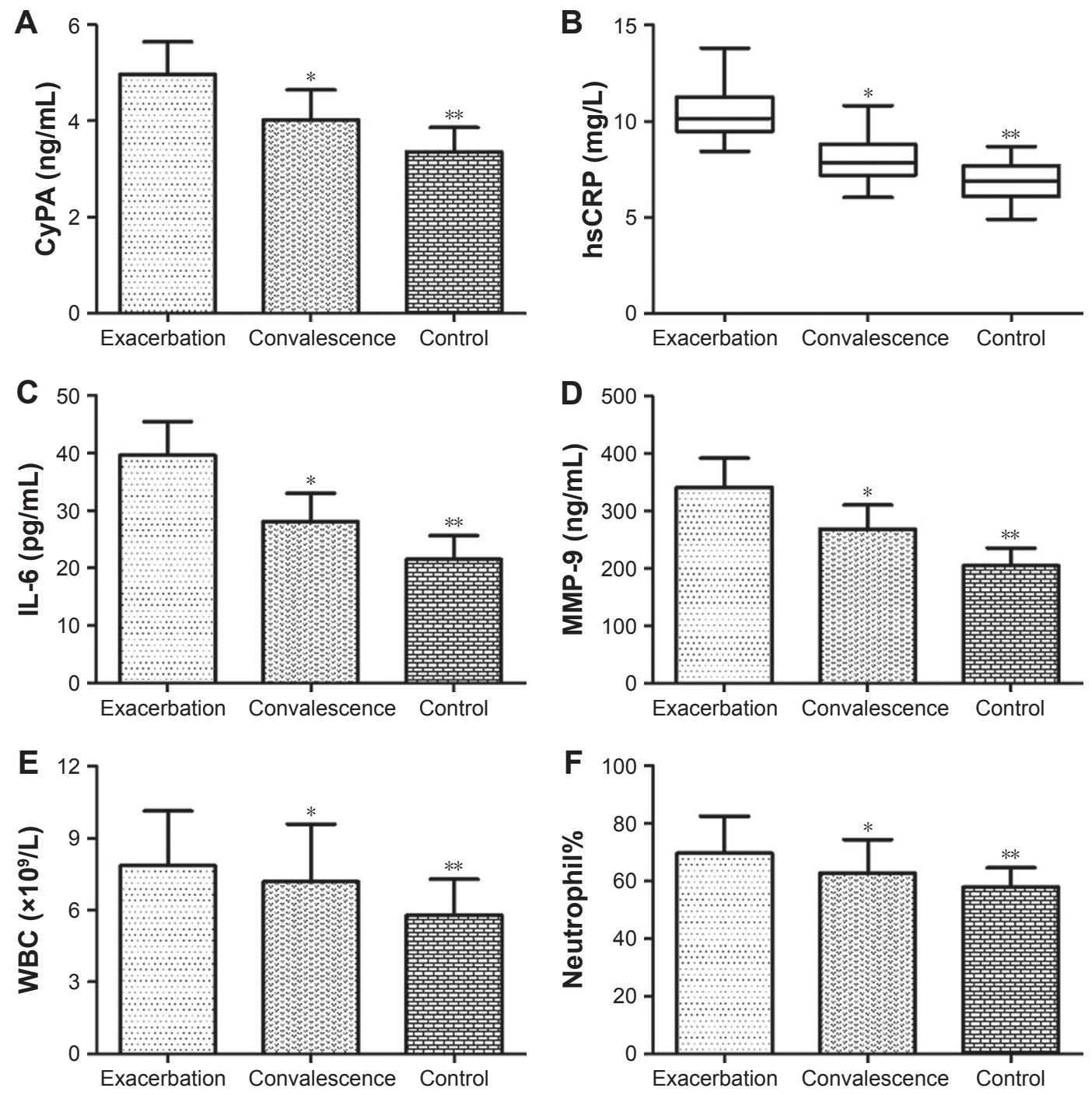

Figure 2 Inflammation indexes in COPD patients with exacerbation and convalescence phases $(n=93)$ and healthy controls ( $n=88)$.

Notes: Serum levels of CyPA (A), hsCRP (B), IL-6 (C) and MMP-9 (D) were measured by enzyme-linked immunosorbent assay, and values of WBC (E) and neutrophil\% (F) were determined by an autoanalyser. $* P<0.05$ versus the exacerbated $C O P D$ patients, and $* * P<0.05$ versus the convalescent $C O P D$ patients.

Abbreviations: COPD, chronic obstructive pulmonary disease; CyPA, cyclophilin A; hsCRP, high-sensitivity C-reactive protein; IL-6, interleukin-6; MMP-9, matrix metalloproteinase-9; WBC, white blood cells.

and thereby initiates a cascade of inflammatory processes such as leucocyte chemotaxis and induction of MMP-9 and IL-6 expression. ${ }^{31,32}$ Therefore, we further evaluated serum concentrations of IL-6 and MMP-9 in COPD patients.

Table 2 Partial correlation coefficients of CyPA with laboratory parameters in exacerbated COPD

\begin{tabular}{lll}
\hline Variables & $\boldsymbol{r}$ & $\boldsymbol{P}$-value \\
\hline hsCRP & 0.442 & $<0.00 \mathrm{I}$ \\
IL-6 & 0.343 & $0.00 \mathrm{I}$ \\
MMP-9 & 0.749 & $<0.001$ \\
WBC & 0.386 & $<0.001$ \\
Neutrophil\% & 0.254 & 0.017 \\
\hline
\end{tabular}

Abbreviations: COPD, chronic obstructive pulmonary disease; CyPA, cyclophilin A; hsCRP, high-sensitivity C-reactive protein; IL-6, interleukin-6; MMP-9, matrix metalloproteinase-9; WBC, white blood cells.
Serum levels of IL-6 and MMP-9 were also increased in convalescent COPD patients and further increased in exacerbated COPD patients. Correlation analysis showed that CyPA positively correlated with IL-6 and MMP-9 in COPD patients with acute exacerbation and convalescence phases, suggesting that CyPA can strengthen its effect through the induction of IL-6 and MMP-9 expression.

COPD is characterized not only by local pulmonary inflammation, but also by systemic inflammation. ${ }^{33}$ Various systemic inflammatory markers, including WBC, neutrophil $\%$, mean platelet volume, erythrocyte sedimentation rate, hsCRP, D-dimer, cystatin C, and inflammatory cytokines have been used for the assessment of inflammation during COPD progression. ${ }^{7,8,34-36}$ This study found that hsCRP, WBC, and neutrophil\% were significantly increased in both 
Table 3 Partial correlation coefficients of CyPA with laboratory parameters in convalescent COPD

\begin{tabular}{lll}
\hline Variables & $\boldsymbol{r}$ & $\boldsymbol{P}$-value \\
\hline hsCRP & $0.44 \mathrm{I}$ & $<0.00 \mathrm{I}$ \\
IL-6 & 0.307 & 0.004 \\
MMP-9 & 0.778 & $<0.00 \mathrm{I}$ \\
WBC & 0.197 & 0.067 \\
Neutrophil\% & 0.164 & 0.128 \\
FVC (\% predicted) & -0.154 & 0.154 \\
FEV (\% predicted) & -0.271 & 0.011 \\
FEV,/FVC & -0.291 & 0.006 \\
\hline
\end{tabular}

Abbreviations: COPD, chronic obstructive pulmonary disease; CyPA, cyclophilin A; hsCRP, high-sensitivity C-reactive protein; IL-6, interleukin-6; MMP-9, matrix metalloproteinase-9; WBC, white blood cells; FVC, forced vital capacity; FEV, forced expiratory volume in I second.

the exacerbation and convalescence phases of COPD, indicating that the inflammatory response persists through the convalescence phase. Correlation analysis showed that serum CyPA positively correlated with hsCRP during the exacerbation and convalescence phases of COPD, and negatively correlated with $\mathrm{FEV}_{1} \%$ predicted and $\mathrm{FEV}_{1} / \mathrm{FVC}$ in the convalescence phase. These results suggested that serum CyPA may be another inflammatory marker for COPD. In addition, the results of multivariate linear regression analysis suggested that age, gender, body mass index, smoking status, course of disease, and complication with hypertension did not significantly affect serum CyPA concentration, and COPD disease status was found to be the only major factor.

The strengths of our present study are the well-matched controls and direct comparison of CyPA at different phases of COPD. As diabetes mellitus and coronary artery disease affect serum CyPA expression, ${ }^{13,14}$ we ruled out these comorbidities in this study. It has also been reported that plasma

Table 4 Multivariate linear regression of COPD patients with CyPA as the dependent variable

\begin{tabular}{lllll}
\hline Variables & $\begin{array}{l}\text { Nonstandard } \\
\text { coefficient }\end{array}$ & $\begin{array}{l}\text { Standard } \\
\text { coefficient }\end{array}$ & t & P-value \\
\hline Constant & 4.759 & & 8.328 & 0.000 \\
Age & 0.008 & 0.072 & 1.112 & 0.268 \\
Gender & 0.138 & 0.072 & 0.895 & 0.372 \\
Body mass index & 0.005 & 0.023 & 0.390 & 0.697 \\
Smoking status & 0.136 & 0.128 & 1.631 & 0.105 \\
Course of disease & 0.000 & -0.010 & -0.162 & 0.871 \\
Hypertension & 0.152 & 0.073 & 1.174 & 0.242 \\
Disease status & -0.954 & -0.591 & -10.186 & 0.000 \\
\hline
\end{tabular}

Notes: Definition of gender: male $=1$, female $=2$; smoking status: current smokers =I, former smokers $=2$, never smokers $=3$; hypertension: presence of hypertension $=\mathrm{I}$, nonpresence of hypertension $=2$; disease status: exacerbated COPD $=1$, convalescent $\mathrm{COPD}=2$.

Abbreviations: COPD, chronic obstructive pulmonary disease; CyPA, cyclophilin A.
CyPA level is influenced by essential hypertension, ${ }^{37}$ so the patients and controls were well matched for hypertension proportion in the present study. Moreover, our results first suggest that serum CyPA may be a potential inflammatory biomarker for COPD. However, we should confirm that there are some limitations in this study. First, treatments of COPD patients may influence the statistical results among the three groups. Second, the pulmonary function of exacerbated COPD patients is absent. Third, the sample size in this study was not large. Finally, our study excluded COPD patients with comorbidities, so the results may not be generalized to all COPD patients.

In conclusion, this study shows that serum CyPA levels were increased in convalescent COPD patients compared to healthy controls, and further elevated in COPD patients with acute exacerbation. Moreover, serum CyPA positively correlated with hsCRP, IL-6, and MMP-9 in COPD with convalescence and exacerbation phases, and negatively correlated with $\mathrm{FEV}_{1} \%$ predicted and $\mathrm{FEV}_{1} / \mathrm{FVC}$ in convalescent COPD patients. Serum CyPA may be a reliable biomarker for assessing the systemic inflammatory response in COPD progression.

\section{Acknowledgments}

This study was supported by the National Nature Science Foundation of China (No 81600030), Natural Science Basic Research Project of Shaanxi Province (No 2016JQ8048), and Research Funds of The Second Affiliated Hospital of Xi'an Jiaotong University (No RC(XM)201604).

\section{Disclosure}

The authors report no conflicts of interest in this work.

\section{References}

1. Lopez-Campos JL, Tan W, Soriano JB. Global burden of COPD. Respirology. 2016;21(1):14-23.

2. Vijayan VK. Chronic obstructive pulmonary disease. Indian J Med Res. 2013;137(2):251-269.

3. Cazzola M, Page CP, Calzetta L, Matera MG. Emerging antiinflammatory strategies for COPD. Eur Respir J. 2012;40(3):724-741.

4. Tetley TD. Inflammatory cells and chronic obstructive pulmonary disease. Curr Drug Targets Inflamm Allergy. 2005;4(6):607-618.

5. Gompertz S, Bayley DL, Hill SL, Stockley RA. Relationship between airway inflammation and the frequency of exacerbations in patients with smoking related COPD. Thorax. 2001;56(1):36-41.

6. Barnes PJ, Celli BR. Systemic manifestations and comorbidities of COPD. Eur Respir J. 2009;33(5):1165-1185.

7. Karadeniz G, Polat G, Senol G, Buyuksirin M. C-reactive protein measurements as a marker of the severity of chronic obstructive pulmonary disease exacerbations. Inflammation. 2013;36(4):948-953.

8. MacNee W. Systemic inflammatory biomarkers and co-morbidities of chronic obstructive pulmonary disease. Ann Med. 2013;45(3): 291-300. 
9. Wang P, Heitman J. The cyclophilins. Genome Biol. 2005;6(7):226.

10. Handschumacher RE, Harding MW, Rice J, Drugge RJ, Speicher DW. Cyclophilin: a specific cytosolic binding protein for cyclosporin A. Science. 1984;226(4674):544-547.

11. Nigro P, Pompilio G, Capogrossi MC. Cyclophilin A: a key player for human disease. Cell Death Dis. 2013;4:e888.

12. Nigro P, Satoh K, O'Dell MR, et al. Cyclophilin A is an inflammatory mediator that promotes atherosclerosis in apolipoprotein E-deficient mice. J Exp Med. 2011;208(1):53-66.

13. Yan J, Zang X, Chen R, et al. The clinical implications of increased cyclophilin A levels in patients with acute coronary syndromes. Clin Chim Acta. 2012;413(7-8):691-695.

14. Ramachandran S, Venugopal A, Kutty VR, et al. Plasma level of cyclophilin A is increased in patients with type 2 diabetes mellitus and suggests presence of vascular disease. Cardiovasc Diabetol. 2014;13:38.

15. Liu MC, Lee YW, Lee PT, et al. Cyclophilin A is associated with peripheral artery disease and chronic kidney disease in geriatrics: the Tianliao Old People (TOP) study. Sci Rep. 2015;5:9937.

16. Vestbo J, Hurd SS, Agusti AG, et al. Global strategy for the diagnosis, management, and prevention of chronic obstructive pulmonary disease: GOLD executive summary. Am J Respir Crit Care Med. 2013;187(4): 347-365.

17. Schlegel J, Armstrong GS, Redzic JS, Zhang F, Eisenmesser EZ. Characterizing and controlling the inherent dynamics of cyclophilin-A Protein Sci. 2009;18(4):811-824.

18. Zhou D, Mei Q, Li J, He H. Cyclophilin A and viral infections. Biochem Biophys Res Commun. 2012;424(4):647-650.

19. Seko Y, Fujimura T, Taka H, Mineki R, Murayama K, Nagai R. Hypoxia followed by reoxygenation induces secretion of cyclophilin A from cultured rat cardiac myocytes. Biochem Biophys Res Commun. 2004;317(1):162-168.

20. Satoh K, Nigro P, Matoba T, et al. Cyclophilin A enhances vascular oxidative stress and the development of angiotensin II-induced aortic aneurysms. Nat Med. 2009;15(6):649-656.

21. Soe NN, Sowden M, Baskaran P, et al. Acetylation of cyclophilin A is required for its secretion and vascular cell activation. Cardiovasc Res. 2014;101(3):444-453.

22. Qu X, Wang C, Zhang J, Qie G, Zhou J. The roles of CD147 and/or cyclophilin A in kidney diseases. Mediators Inflamm. 2014;2014:728673.

23. Satoh K, Shimokawa H, Berk BC. Cyclophilin A: promising new target in cardiovascular therapy. Circ J. 2010;74(11):2249-2256.
24. Wang L, Wang CH, Jia JF, et al. Contribution of cyclophilin A to the regulation of inflammatory processes in rheumatoid arthritis. $J$ Clin Immunol. 2010;30(1):24-33.

25. Tegeder I, Schumacher A, John S, et al. Elevated serum cyclophilin levels in patients with severe sepsis. J Clin Immunol. 1997;17(5):380-386.

26. Lee J. Role of cyclophilin a during oncogenesis. Arch Pharm Res. 2010;33(2):181-187.

27. Avramut M, Achim CL. Immunophilins in nervous system degeneration and regeneration. Curr Top Med Chem. 2003;3(12):1376-1382.

28. Stemmy EJ, Balsley MA, Jurjus RA, Damsker JM, Bukrinsky MI, Constant SL. Blocking cyclophilins in the chronic phase of asthma reduces the persistence of leukocytes and disease reactivation. Am J Respir Cell Mol Biol. 2011;45(5):991-998.

29. Liu L, Li C, Xiang J, Dong W, Cao Z. Over-expression and potential role of cyclophilin A in human periodontitis. J Periodontal Res. 2013; 48(5):615-622.

30. Hu R, Ouyang Q, Dai A, Tan S, Xiao Z, Tang C. Heat shock protein 27 and cyclophilin A associate with the pathogenesis of COPD. Respirology. 2011;16(6):983-993.

31. Yurchenko V, Zybarth G, O'Connor M, et al. Active site residues of cyclophilin A are crucial for its signaling activity via CD147. J Biol Chem. 2002;277(25):22959-22965.

32. Yuan W, Ge H, He B. Pro-inflammatory activities induced by CyPAEMMPRIN interaction in monocytes. Atherosclerosis. 2010;213(2): 415-421.

33. Barnes PJ. Cellular and molecular mechanisms of chronic obstructive pulmonary disease. Clin Chest Med. 2014;35(1):71-86.

34. Zhang M, Li Y, Yang X, et al. Serum cystatin c as an inflammatory marker in exacerbated and convalescent COPD patients. Inflammation. 2016;39(2):625-631.

35. Zhang M, Li Y, Zhang J, et al. Mean platelet volume is elevated in exacerbated and convalescent COPD patients. Clin Chim Acta. 2015; 451(Pt B):227-231.

36. Zhang M, Zhang J, Zhang Q, et al. D-dimer as a potential biomarker for the progression of COPD. Clin Chim Acta. 2016;455:55-59.

37. Chang CS, Su SL, Chang CC, et al. Cyclophilin-A: a novel biomarker for untreated male essential hypertension. Biomarkers. 2013;18(8): 716-720.
International Journal of COPD

\section{Publish your work in this journal}

The International Journal of COPD is an international, peer-reviewed journal of therapeutics and pharmacology focusing on concise rapid reporting of clinical studies and reviews in COPD. Special focus is given to the pathophysiological processes underlying the disease, intervention programs, patient focused education, and self management protocols.

\section{Dovepress}

This journal is indexed on PubMed Central, MedLine and CAS. The manuscript management system is completely online and includes a very quick and fair peer-review system, which is all easy to use. Visit http://www.dovepress.com/testimonials.php to read real quotes from published authors. 